\title{
Effects of Surface Diffusion and Heating Rate on First Stage Sintering That Densifies by Grain-boundary Diffusion
}

\author{
Wendong Luo and Jingzhe Pan* \\ Department of Engineering, University of Leicester, \\ Leicester, LE1 7RH, United Kingdom
}

\begin{abstract}
This paper presents a computational study of the role played by surface diffusion on first stage sintering of powders that densify by grain-boundary diffusion. Coupled grain-boundary and surface diffusion is considered as the mechanism for matter redistribution. By using several novel approaches of presentation of the numerical data, it is shown that the assumption of fast surface diffusion is invalid for typical sintering conditions and materials in first stage of sintering. The study reveals a simple explanation for the role played by surface diffusion in matter redistribution of combined grain-boundary and surface diffusion - surface diffusion changes direction from moving atoms away from a contact neck to depositing atoms onto it as the rate of surface diffusion increases. The reverse surface diffusion blunts the neck and retards densification. It is shown that this mechanism is significant not only for free sintering but also for pressure assisted sintering. It is further confirmed that the widely observed beneficial effect of spark plasma sintering on densification can be, at least partially, attributed to its fast heating rate, which quickly passes through sintering at low temperatures where surface diffusion dominates.
\end{abstract}

* Corresponding author, jp165@le.ac.uk

\section{Introduction}

Surface diffusion is an important mechanism for matter redistribution. In some powder systems under certain conditions, sintering can occur by surface diffusion alone. Kuczynski ${ }^{1}$ This paper was presented at SINTERING 2014, Dresden, Germany. 
considered various matter transportation mechanisms for sintering of metallic powders. However powder compact of most ceramics sinters by a combination of grain-boundary and surface diffusion. In this sintering mechanism, grain-boundary diffusion at the particle contacts is responsible for densification. The role played by surface diffusion is however rather complex. It is often argued that surface diffusion is much faster than grain-boundary diffusion, and hence materials delivered by grain-boundary diffusion to the contact neck can be quickly redistributed onto the pore surface by surface diffusion. It follows that grainboundary diffusion is the rate limiting process. Many analytical models for sintering from the classical model by Coble ${ }^{2}$ to modern constitutive laws ${ }^{3,4}$, are based on this assumption of fast surface diffusion. While this assumption is valid for later stage of sintering when pores are isolated, it has been generally recognised that surface diffusion plays an important role in the first stage of sintering when the contacts between particles are isolated. Earlier works on this topic includes those by Nichols and Mullins ${ }^{5}$, German and Lathrop ${ }^{6}$, Bross and Exner ${ }^{7}$ and Exner $^{8}$. In particular the simulation results of Exner ${ }^{7}$ showed that surface redistribution from the grain boundary would limit grain boundary diffusion only for high grain boundary diffusivity. Svoboda and Riedel ${ }^{9}$ developed an analytical treatment for surface diffusion to model its effect on sintering behaviour. Bouvard and McMeeking ${ }^{10}$ provided an analytical contact law between particles by fitting their numerical results of computer simulation of sintering by coupled surface and grain-boundary diffusion. Bouvard and McMeeking demonstrated that fast surface diffusion accelerates neck growth and retards shrinkage. One technical issue for modelling coupled surface and grain-boundary diffusion is how to calculate the curvature at the triple junction between the grain-boundary and particle surfaces. The curvature is required for both calculating surface diffusion flux and completing the solution for grain-boundary diffusion. It however does not exist mathematically because of tangential discontinuity of the free surface at the junction. Pan and Cocks ${ }^{11}$ resolved the issue 
by showing that it is possible to find an expression for the chemical potential at the triple junction which should be used in the calculation instead of the curvature. Zhang and Gladwell ${ }^{12}$ studied the effect of surface diffusion on sintering of particles of different sizes and showed that careful treatment of the triple junction is important when modelling the sintering process. There are many immediate and important applications of studying the effect of surface diffusion. Chu et $a l^{13}$ were probably the first to highlight the effect of surface diffusion on practical sintering practice. Because surface diffusion tends to dominate over grain-boundary diffusion at lower temperatures, it may be possible to attribute, at least partially, the fast densification rates achieved by Spark Plasma Sintering (SPS) to the fast heating rate. Olevsky et $a l^{14}$ developed a constitutive model considering the interplay between three mechanisms of material transport: surface diffusion, grain-boundary diffusion and power-law creep. Their model predicts that a better sinterability can be achieved by increasing the heating rate and this effect is attributed to the different shapes that the pore develops under different heating rates.

Despite all the existing understanding about the important role played by surface diffusion, the effect of surface diffusivity is often ignored in sintering models particularly in many studies of first stage sintering using the discrete element method. This is mainly due to two reasons. Firstly the problem of surface diffusion has to be solved numerically in general. It is difficult to build the numerical solution into a sintering model for powder compact made of millions of three dimensional particles. The assumption of fast surface diffusion simplifies the modelling effort considerably. However recent development of combining sintering models at continuum and particle levels ${ }^{15}$ has made it possible to take into account of surface diffusion fully in finite element analysis of sintering at the component scale. Secondly the assumption seems plausible. The effect of surface diffusion, although well understood by many previous researchers, was buried in analytical expressions and numerical details. A 
simple demonstration, in particular relating to experimental data, on the effect of surface diffusion in densification dominated by grain-boundary diffusion is missing.

The purpose of this paper is to (a) present a simple and definitive case for invalidity of the assumption of fast surface diffusion for stage one sintering where densification is achieved by grain-boundary diffusion, (b) provide a straightforward understanding in why fast surface diffusion retards densification under such conditions and (c) reconfirmed the work by Olevsky et $a l^{14}$ to explain how fast heating rate favours densification. The co-sintering problem of two spherical particles is revisited using the numerical method developed by Pan and Cocks ${ }^{11}$. One tool used in this demonstration is to examine the relative relation between the shrinkage and neck growth as sintering proceeds. A small ratio of shrinkage over neck growth indicates that the sintering process is detrimental to densification. The ratio is zero if grain-boundary diffusion is completely prohibited. On the other hand a large ratio indicates that the sintering process favours densification. Another tool to demonstrate the effect of surface diffusion is to examine the direction of the surface diffusion flux in the vicinity of the triple junction between grain-boundary and particle surfaces. If the flux is away from the junction region, then surface diffusion is helping densification by moving atoms away and depositing them onto pore surfaces. If the flux is towards the junction region, then surface diffusion is harming the densification by moving atoms from the particle surfaces onto the junction region and reducing the driving force for further densification. These tools were available to all previous researchers who developed numerical models but have not been used to make the case for the effect of surface diffusion as far as we are aware.

\section{Governing Equations and their Numerical Solution}

We consider two identical and spherical particles as shown in Fig. 1(a) which sinter by coupled surface and grain-boundary diffusion. In the figure $\mathrm{R}$ represents the initial radius of 
two particles, 2a the neck diameter, $\gamma_{\mathrm{s}}$ the specific surface energy, $\gamma_{\mathrm{gb}}$ the specific grainboundary energy and $\theta$ the dihedral angle at the triple junction. A cylindrical coordinates (r, z) are used. The governing equations used here are identical to those used by Svoboda and Riedel $^{9}$ and by Bouvard and McMeeking ${ }^{10}$ and therefore only briefly introduced.

According to Fick's law, the diffusive flux $\mathrm{j}$ is linearly dependent on the gradient of the chemical potential $\mu$ of the diffusion atoms such that

$$
\mathrm{j}=-\frac{\mathrm{D} \delta}{\mathrm{kT}} \frac{\partial \mu}{\partial \mathrm{s}}
$$

where $\mathrm{D}$ is the diffusivity, $\delta$ the thickness of diffusion layer, $\mathrm{k}$ the Boltzmann constant, $\mathrm{T}$ the sintering temperature and s the local coordinate along the diffusion path. On the grainboundary, the chemical potential is related to the stress $\sigma$ normal to grain boundary through

$$
\mu=-\Omega \sigma
$$

where $\Omega$ is the atomic volume. Substituting Eq. (2) into Eq. (1) leads to

$$
\mathrm{j}_{\mathrm{gb}}=\mathcal{D}_{\mathrm{gb}} \frac{\partial \sigma}{\partial \mathrm{r}}
$$

in which an effective grain-boundary diffusivity has been introduced as

$$
\mathcal{D}_{\mathrm{gb}}=\frac{\mathrm{D}_{\mathrm{gb}} \delta_{\mathrm{gb}} \Omega}{\mathrm{kT}}
$$

where $\mathrm{D}_{\mathrm{gb}}$ is grain-boundary diffusivity and $\delta_{\mathrm{gb}}$ is thickness of grain-boundary diffusion layer. Using matter conservation and Eq. (3), the expressions for the grain-boundary flux $\mathrm{j}_{\mathrm{gb}}$ and normal stress $\sigma$ can be found as ${ }^{10}$

$$
\mathrm{j}_{\mathrm{gb}}=-\frac{1}{2} V_{g b} * \mathrm{r}
$$


and

$$
\sigma(r)=-\frac{1}{4 \mathcal{D}_{\mathrm{gb}}} \mathrm{V}_{\mathrm{gb}} \mathrm{r}^{2}+\mathrm{C}
$$

in which $V_{g b}$ represents the approaching velocity between the two particles and $C$ is an integration constant to be determined.

On the particle surface, the chemical potential is related to the principal curvature $\kappa$ through

$\mu=-\Omega \gamma_{\mathrm{s}} \kappa$

Substituting Eq. (7) into Eq. (1) leads to

$$
\mathrm{j}_{\mathrm{s}}=\mathcal{D}_{\mathrm{s}} \frac{\partial \kappa}{\partial \mathrm{s}}
$$

in which an effective surface diffusivity has been introduced as

$\mathcal{D}_{\mathrm{s}}=\frac{\mathrm{D}_{\mathrm{s}} \delta_{\mathrm{s}} \Omega \gamma_{\mathrm{s}}}{\mathrm{kT}}$

where $\mathrm{D}_{\mathrm{s}}$ is surface diffusivity and $\delta_{s}$ is thickness of diffusion layer. Matter conservation provides a relation between the surface migration velocity $V_{s}$ and the surface diffusion flux $\mathrm{j}_{\mathrm{s}}$ such that

$$
\mathrm{V}_{\mathrm{s}}+\frac{\partial \mathrm{j}_{\mathrm{s}}}{\partial \mathrm{s}}=0
$$

Away from the triple junction, Eqs. (8) and (10) can be solved using the finite difference method by Bouvard and McMeeking ${ }^{10}$.

The grain-boundary stress $\sigma(r)$, the surface tension $\gamma_{s}$ and the remotely applied stress $\sigma_{\infty}$ must satisfy mechanical equilibrium such that 


$$
\int_{0}^{a} \sigma(r) 2 \pi r d r=\sigma_{\infty} \pi \mathrm{a}^{2}-\gamma_{\mathrm{s}} * 2 \pi \mathrm{a} * \sin \theta
$$

At the triple junction the surface tension $\gamma_{\mathrm{s}}$ and grain-boundary tension $\gamma_{\mathrm{gb}}$ must be in equilibrium which leads to

$$
\cos \theta=\frac{\gamma_{\mathrm{gb}}}{2 \gamma_{\mathrm{s}}}
$$

The equilibrium dihedral angle has to be enforced when updating the profiles of the free surface at the triple junction. At the junction matter conservation requires that

$\mathrm{j}_{\mathrm{gb}}-2 \mathrm{j}_{\mathrm{s}}=0$

Furthermore the chemical potential must be continuous from the grain-boundary to the particle surface which leads to

$$
\mu_{\text {tip }}=-\Omega \sigma_{\text {tip }}=-\Omega \gamma_{\mathrm{s}} \kappa_{\text {tip }} \Rightarrow \sigma_{\text {tip }}=\gamma_{\mathrm{s}} \kappa_{\text {tip }}
$$

Mathematically the principal curvature $\kappa_{\text {tip }}$ is not defined at the triple junction and cannot be calculated using any finite difference scheme because of the tangential discontinuity of the free surface. However the chemical potential $\mu_{\text {tip }}$ does exist at the triple junction and a nominal $\kappa_{\text {tip }}$ can be defined accordingly using Eq. (13).

The above set of equations contain three unknowns to be determined including $\mathrm{V}_{\mathrm{gb}}$, $\mathrm{C}$ and $\kappa_{\text {tip }}$. Pan and Cocks ${ }^{11}$ demonstrated that these parameters can be uniquely determined using Eqs (11), (13) and (14) together with a finite difference scheme for the surface diffusion. Pan and Cocks ${ }^{11}$ provided a general treatment of the problem. Here some details are provided to show how their general concept can be applied to the simple problem of two particle sintering. As shown in Fig. 1(b), the surface flux at mid-point 1 close to the triple junction can be calculated using a finite difference version of Eq. (8), i.e. we have This paper was presented at SINTERING 2014, Dresden, Germany. 
$\mathrm{j}_{\mathrm{s} 1}=\mathcal{D}_{\mathrm{s}} \frac{\kappa_{1}-\kappa_{\mathrm{tip}}}{\Delta \mathrm{S}_{1}}$

in which $\kappa_{1}$ is the principal curvature of finite difference node 1 . The principal curvature $\kappa_{1}$ consists of two parts with one part calculated by drawing a circle through nodes 1,2 and the junction point and the other part from the distance between node 1 to the vertical axis z. In Eq. (15) $\Delta S_{1}$ is the arc length between node 1 and the junction. Using $r=a$ at the junction Eq. (6) becomes

$$
\sigma_{t i p}=-\frac{1}{4 \mathcal{D}_{\mathrm{gb}}} \mathrm{V}_{\mathrm{gb}} \mathrm{a}^{2}+\mathrm{C}
$$

Combining Eq. (14) and (16), we have

$$
-\frac{1}{4 \mathcal{D}_{\mathrm{gb}}} \mathrm{V}_{\mathrm{gb}} \mathrm{a}^{2}+\mathrm{C}=\gamma_{\mathrm{s}} \kappa_{\mathrm{tip}}
$$

Substituting Eq. (6) into Eq. (11) and combining Eq. (17), we have

$$
V_{g b}=8 \mathcal{D}_{\mathrm{gb}}\left(\frac{\sigma_{\infty}}{a^{2}}-\frac{2 \gamma_{\mathrm{s}} \sin \theta}{a^{3}}-\frac{\gamma_{\mathrm{s}} \kappa_{\mathrm{tip}}}{a^{2}}\right)
$$

Substituting Eq. (5) and (15) into Eq. (13) and noticing that $r=a$ at the junction lead to

$$
\mathrm{V}_{\mathrm{gb}}=-\frac{4 \mathcal{D}_{\mathrm{s}}}{\mathrm{a}}\left(\frac{\kappa_{1}-\kappa_{\mathrm{tip}}}{\Delta \mathrm{S}_{1}}\right)
$$

Equating the right hand side of Eq (18) with that of Eq. (19) provides a equation from which $\kappa_{\text {tip }}$ can be found as

$$
\kappa_{\text {tip }}=\left[2 \mathcal{D}_{\mathrm{gb}}\left(\frac{\sigma_{\infty}}{a}-\frac{2 \gamma_{\mathrm{s}} \sin \theta}{a^{2}}\right)+\mathcal{D}_{\mathrm{s}}\left(\frac{\kappa_{1}}{\Delta \mathrm{S}_{1}}\right)\right] /\left(\frac{2 \mathcal{D}_{\mathrm{gb}} \gamma_{\mathrm{s}}}{a}+\frac{\mathcal{D}_{\mathrm{s}}}{\Delta \mathrm{S}_{1}}\right) .
$$

Then using Eq. (18) and (20) in Eq. (17) gives 
$C=2 \sigma_{\infty}-\frac{4 \gamma_{s} \sin \theta}{a}-\gamma_{s} \kappa_{t i p}$

It can be observed from Eq. (20) that $\kappa_{\text {tip }}$ depends on the diffusivities, the remote stress and the specific surface energy. The effective diffusivities $\mathcal{D}_{\text {gb }}$ and $\mathcal{D}_{\mathrm{s}}$ depend on temperature such that

$\mathcal{D}_{\mathrm{gb}}=\frac{\Omega\left(\mathrm{D}_{\mathrm{gb} 0} \delta_{\mathrm{gb}}\right) \exp \left(-\mathrm{Q}_{\mathrm{gb}} / \mathrm{RT}\right)}{\mathrm{kT}}$

and

$\mathcal{D}_{\mathrm{s}}=\frac{\Omega \gamma_{\mathrm{s}}\left(\mathrm{D}_{\mathrm{s} 0} \delta_{\mathrm{s}}\right) \exp \left(-\mathrm{Q}_{\mathrm{s}} / \mathrm{RT}\right)}{\mathrm{kT}}$

in which $\mathrm{D}_{\mathrm{gb} 0}$ and $\mathrm{D}_{\mathrm{s} 0}$ are the pre-exponential coefficients for the grain-boundary and surface diffusivities respectively, $\mathrm{Q}_{g b}$ and $\mathrm{Q}_{s}$ are the activation energies of grain-boundary and surface diffusion respectively and $\mathrm{R}$ is the gas constant. In this study the thickness of surface diffusion layer $\delta_{\mathrm{s}}$ is taken as $\sqrt[3]{\Omega}$.

\section{Effect of surface diffusion on neck growth and shrinkage}

Table I presents the material data used in this study which are loosely based on micron-sized alumina powders ${ }^{16}$. The isothermal sintering temperature is selected as $1200^{\circ} \mathrm{C}$. Using the data in Table I and the sintering temperature, Eq. (22) gives $\mathcal{D}_{\text {gb }}=6.853 \times 10^{-36} \mathrm{~m}^{4} \mathrm{~s} / \mathrm{kg}$. The effective surface diffusivity $\mathcal{D}_{\text {s }}$ was varied from 5 times to 10000 times of $\mathcal{D}_{\text {gb }}$ to explore the effect of surface diffusion. When presenting the results, the time is normalised by a characteristic time defined as $\tau_{g}=k T R^{4} / \delta_{g b} D_{g b} \Omega \gamma_{s}{ }^{10}$, which is time for the neck to reach $\mathrm{R}$ by grain-boundary diffusion ${ }^{17}$. All the simulations started from a small initial contact area of $\mathrm{a} / \mathrm{R}=0.01$. 


\section{(1) Neck growth}

Fig. 2 shows the time taken for the neck radius to reach a $=0.25 \mathrm{R}$ as a function of the relative diffusivity $\mathcal{D}_{\mathrm{s}} / \mathcal{D}_{\text {gb }}$ for different values of the remote stress $\sigma_{\infty}$. Three observations can be made from the figure:

a) a faster surface diffusion always leads to a faster neck growth over this large range of $\mathcal{D}_{\mathrm{s}} / \mathcal{D}_{\mathrm{gb}}$

b) the neck growth rate is very sensitive to $\mathcal{D}_{\mathrm{s}} / \mathcal{D}_{\text {gb }}$ and therefore the assumption of fast surface diffusion does not make sense when calculating neck growth at the first stage of sintering and

c) the effect of the applied pressure on neck growth can be ignored if $\mathcal{D}_{\mathrm{s}} / \mathcal{D}_{\text {gb }}>100$.

While these understandings are known from previous analytical and numerical studies, our work provides a straightforward insight into the role played by surface diffusing. Table II presents the directions of the surface diffusion flux at a point that is a small distance away from the triple junction. In these cases the applied external pressure is set as zero. The observation point is selected at a small arc length of $\mathrm{s} / \mathrm{R}=0.06$ away from the triple junction. In the numerical analysis, the surface diffusion flux may oscillate with time. In order to obtain a stabled direction, the surface diffusion flux at the observation point was integrated over a small time period of $t=10^{-9} \tau_{g}$. It can be seen from the Table that surface diffusion changes direction at $\mathcal{D}_{\mathrm{s}} / \mathcal{D}_{\mathrm{gb}}=50$. If surface diffusion is not too fast relative to grainboundary diffusion, it helps to move atoms away from the triple junction. However if surface diffusion is about 50 times faster than grain-boundary diffusion, it moves atoms from the particle surface unto the small region near the junction. This does not mean that boundary condition (13) is violated or that surface diffusion feeds material into the grain-boundary. In the situation where surface diffusion moves atoms from the particle surface unto the junction 
region, grain-boundary diffusion also moves atoms from the grain-boundary unto the junction region. This result has a profound implication on our understanding for the role played by surface diffusion on the sintering process. Figs 3 (a) and (b) compare the profiles of the triple junction for $\mathcal{D}_{\mathrm{s}} / \mathcal{D}_{\mathrm{gb}}=5$ and $\mathcal{D}_{\mathrm{s}} / \mathcal{D}_{\mathrm{gb}}=50$ respectively at two different times. For $\mathcal{D}_{\mathrm{s}} /$ $\mathcal{D}_{\mathrm{gb}}=50$ because of the revers surface diffusion, the neck grows faster than that for $\mathcal{D}_{\mathrm{s}} /$ $\mathcal{D}_{\mathrm{gb}}=5$. However the local curvature near the junction also becomes smaller for $\mathcal{D}_{\mathrm{s}} / \mathcal{D}_{\mathrm{gb}}=$ 50 than in the case of $\mathcal{D}_{\mathrm{s}} / \mathcal{D}_{\mathrm{gb}}=5$. As shown in the next sub-section, this blunting of the triple junction retards grain-boundary diffusion and hence slows down densification.

Fig. 4 and 5 provide the neck-size as a function of time for $\mathcal{D}_{\mathrm{s}} / \mathcal{D}_{\text {gb }}=5$ and 50 under a range of remote compressive stresses 0-100MPa. These are the typical way in which results are presented in computer simulations of sintering. It is rather difficult to examine these figures because too much information is presented. These figures are provided here for completeness.

\section{(2) Shrinkage}

Fig. 6 shows the time taken for the shrinkage to reach $w=0.01 \mathrm{R}$ as a function of $\mathcal{D}_{\mathrm{s}} / \mathcal{D}_{\mathrm{gb}}$ for a range of remote stress $\sigma_{\infty}$. Three observations can be made from the figure:

a) a faster surface diffusion always leads to a slower shrinkage in opposite to the case for neck growth,

b) the assumption of fast surface diffusion cannot be valid because a change in $\mathcal{D}_{\mathrm{s}} / \mathcal{D}_{\mathrm{gb}}$ always leads to a significant change in the shrinkage rate especially for free sintering,

c) remote pressure always has a significant beneficial effect on neck growth.

Bouvard and McMeeking ${ }^{10}$ reported the detrimental effect of surface diffusion on shrinkage. Our results confirm and strengthen their conclusion using a clearer presentation. Our study also provides a straightforward explanation for this detrimental effect - the surface diffusion This paper was presented at SINTERING 2014, Dresden, Germany. 
changes its direction as $\mathcal{D}_{\mathrm{s}} / \mathcal{D}_{\mathrm{gb}}$ increases. As shown in section III(1), the reversed surface diffusion blunts the triple junction and hence reduces the driving force for grain-boundary diffusion. It is important to observe from Fig. 6 that the detrimental effect of surface diffusion on densification is still significant even if a remote pressure is applied although this effect much weakened. It will be shown in the following section that the blunting effect is important even if a large pressure is applied when examining the effect of heating rate.

Figs. 7 and 8 provide the shrinkage as a function of time for $\mathcal{D}_{\mathrm{s}} / \mathcal{D}_{\mathrm{gb}}=5$ and 50 under a range of remote stresses $0-100 \mathrm{MPa}$. Again it is rather difficult to examine these figures because too much information is presented. These figures are provided here for completeness.

\section{Effect of heating rate on sintering kinetics}

Olevsky et $a l^{14}$ developed a model that predicts increasing heating rate benefits densification because a favourable pore shape develops under higher heating rate. In their model surface diffusion is treated in isolation which is a simplification that requires some validation. Here we present our study following the same argument but using a full numerical analysis. The computer simulation results are presented for sintering under increasing temperature from $600{ }^{\circ} \mathrm{C}$ to $1400{ }^{\circ} \mathrm{C}$ with different heating rates. The effective surface and grain-boundary diffusivities vary with temperature according to Eqs. (22) and (23). Table III presents the data

used for $\mathrm{D}_{\mathrm{gb} 0} \delta_{\mathrm{gb}}$ and $\mathrm{Q}_{g b}$ in the simulations. Table IV presents two sets of data for $\mathrm{D}_{\mathrm{s} 0}$ and $\mathrm{Q}_{s}$ used in the simulations. Data for surface diffusivity reported in the literature varies significantly. In this study two sets of different data of $\mathrm{D}_{\mathrm{s} 0}$ and $\mathrm{Q}_{s}$ are used. These data are loosely based on alumina powders provided in a data book on alumina ${ }^{18}$ and are selected here because they come from the single source. Other parameters used in the numerical study are provided in Table I. 
Fig. 9 shows the values of $\mathcal{D}_{\mathrm{gb}}$ and $\mathcal{D}_{\mathrm{s}}$ as function of temperature. It is clear that $\mathcal{D}_{\mathrm{gb}}$ and $\mathcal{D}_{\mathrm{s}}$ have very different dependences on the temperature because of their different activation energies. At $600^{\circ} \mathrm{C}, \mathcal{D}_{\mathrm{s}}$ is about 4 orders of magnitude larger than $\mathcal{D}_{\text {gb }}$ while at $1400^{\circ} \mathrm{C}, \mathcal{D}_{\mathrm{s}}$ is only about 10 times larger than $\mathcal{D}_{\text {gb }}$ according to data set 2 or even slightly smaller than $\mathcal{D}_{\text {gb }}$ according data set 1 . As observed in section III, this change in the relative diffusivity can have a profound effect on the sintering kinetics. However at lower temperatures the absolute diffusion rates are also several orders of magnitude smaller than those at the higher temperatures. It is therefore difficult to draw conclusions by just observing Fig. 9 alone.

Computer simulations are carried out using heating rates of $2{ }^{\circ} \mathrm{C} / \mathrm{min}$ and $10{ }^{\circ} \mathrm{C} / \mathrm{min}$ which are typical for traditional sintering ${ }^{13}$, and using $100{ }^{\circ} \mathrm{C} / \mathrm{min}$ and $200{ }^{\circ} \mathrm{C} / \mathrm{min}$ which can be achieved by SPS $^{19}$. It is difficult to compare results for the different heating rates because they all have very different time scales of sintering. To eliminate the effect of time scale so that different heating rates can be compared on a single standard, Fig. 10 presents shrinkage versus neck-size as the temperature is increased from $600{ }^{\circ} \mathrm{C}$ to $1400{ }^{\circ} \mathrm{C}$ with the different heating rates. In this case, the applied pressure is set as zero and surface diffusivity of set 1 in Table IV was used. It can be observed from the figure that higher heating rate clearly benefits densification. Comparing the two extreme cases of heating rates of $2{ }^{\circ} \mathrm{C} / \mathrm{min}$ and $200{ }^{\circ} \mathrm{C} / \mathrm{min}$, at any fixed value of neck-size, the fast heating rate generates much more shrinkage than the slow one. This is simply because the faster heating rate quickly passes the low temperature range where surface diffusion is much faster than grain-boundary diffusion and hence minimises the blunting effect.

One would expect that this effect of heating rate diminishes if a remote pressure is applied. Such expectation comes from the proposition that a large remote pressure, relative to the sintering potential for free sintering (which is in the order of 1-2MPa for micron-sized 
powders), would dominate densification and hence the blunting effect becomes less significant. Fig. 11 shows the numerical results for $\sigma_{\infty}=-50 \mathrm{MPa}$ with all other conditions identical to the case of Fig 10. Obviously the applied pressure leads to a larger shrinkage. It is interesting to observe that heating rate effect is still very significant even for such a large pressure. The range of heating rates and high pressure in Fig. 11 covers the entire range from traditional sintering to SPS. It is therefore reasonable to argue that limiting the reverse surface diffusion and its corresponding blunting effect can at least partially explained the SPS effect reported in the literature.

Fig. 12 and 13 present similar results using data set 2 of surface diffusivity in Table IV. The same effects of heating rates are observed. However the shrinkage achieved at a fixed value of neck size is significantly reduced when comparing with Figs. 10 and 11. It can be seen from Fig. 9 that the effective surface diffusivity calculated from data set 2 is higher than that from data set 1 . This faster surface diffusion rate across the temperature range has led to the significant reduction in shrinkage when comparing Fig. 12 and 13 with Fig. 10 and 11. These results once again highlight the important role played by surface diffusion when understanding the effect of heating rate. By examining Fig 13, it can be confirmed that surface diffusion plays the significant role even when a large pressure is applied.

\section{Conclusion}

Our full numerical study reconfirms the following understandings that are known to the sintering literature:

- the assumption of fast surface diffusion is invalid for stage one sintering of typical ceramic powders under practical sintering conditions;

- a faster surface diffusion always leads to a slower shrinkage rate and a faster neck growth rate; 
- the effect of applied pressure on neck growth can be ignored if surface diffusion is fast enough;

- high heating rate is beneficial to densification and can be explained by the role played by surface diffusion - a faster heating rate quickly passes the low temperature range where surface diffusion is fast.

This study reveals a very simple explanation for the effect of surface diffusion. Surface diffusion in the vicinity of the triple junction changes direction from moving atoms away from the junction to depositing atoms onto a small region near the junction as the rate of surface diffusion increases. The reverse surface diffusion blunts the triple junction and hence retards densification. This mechanism is significant even if a pressure, much larger than the sintering potential, is applied. The heating rates and remote pressures used in the study cover the entire range of traditional sintering and SPS. The beneficial effect of SPS can be at least partially attributed to the fast heating rates made possible by the technology.

\section{References}

${ }^{1}$ G.C. Kuczynski, “The mechanism of densification during sintering of metallic particles,” Acta Metall., 4, 58 - 61 (1949).

${ }^{2}$ R. L. Coble, “Initial Sintering of Alumina and Hematite,” J. Am. Ceram. Soc., 41 [2] 55-62 (1958).

${ }^{3}$ J. Pan and A. C. F Cocks, “A Constitutive Model for Stage 2 Sintering of Fine Grained Materials-I. Grain-boundaries Act as Perfect Sources and Sinks for Vacancies”, Acta Metall. Matter., 42 [4] 1215-1222 (1994).

${ }^{4}$ R. M. McMeeking and L. T. Kuhn, “A Diffusional Creep Law for Powder Compacts”, Acta Metall. Matter., 40 [5] 961-969 (1992). 
${ }^{5}$ F. A. Nichols and W. W. Mullins, "Morphological Changes of a Surface of Revolution Due to Capillarity-Induced Surface Diffusion”, J. Appl. Phys., 36 [6] 1826-1835 (1965).

${ }^{6}$ R. M. German and J. F. Lathrop, "Simulation of Spherical Powder Sintering by Surface Diffusion”, J. Mater. Sci., 13 [5] 921-929 (1978).

${ }^{7}$ P. Bross and H. E. Exner, “Computer Simulation of Sintering Processes,” Acta Metall., 27, 1013-1020 (1979).

${ }^{8}$ H. E. Exner, “Neck Shape and Limiting GBD/SD Ratios in Solid State Sintering," Acta Metall., 35 [3] 587-591 (1987).

${ }^{9}$ J. Svoboda and H. Riedel, “New Solutions Describing The Formation of Interparticle Necks in Solid-state Sintering,” Acta Metall. Matter., 43 [1] 1-10 (1995).

${ }^{10}$ D. Bouvard and R. M. McMeeking, "Deformation of Interparticle Necks by Diffusioncontrolled Creep,” J. Am. Ceram. Soc., 79 [3] 666-672 (1996).

${ }^{11}$ J. Pan, and A. C. F. Cocks, “A Numerical Technique for The Analysis of Coupled Surface and Grain-boundary Diffusion,” Acta Metall. Mater., 43 [4] 1395-1406 (1995).

${ }^{12} \mathrm{~W}$. Zhang and I. Gladwell, "Sintering of Two Particles by Surface and Grain Boundary Diffusion - A Three-dimensional Model and A Numerical Study,” Comput. Mater. Sci., 12 [2] 84-104 (1998).

${ }^{13}$ M. Chu, M. N. Rahaman, L. C. D. Jonghe and R. J. Brook, "Effect of heating rate on sintering and coarsening,” J. Am. Ceram. Soc., 74 [6] 1217-1225 (1991).

${ }^{14}$ E. A. Olevsky, S. Kandukuri and L. Froyen, “Consolidation enhancement in spark-plasma sintering: Impact of high heating rates,” J. Appl. Phys., 102 [11] 114913-114924 (2007) 
${ }^{15}$ A. Maximenko, A. Kuzmov, E. Grigoyev and E. Olevsky, “Direct Multi-scale Modeling of Sintering,” J. Am. Ceram. Soc., 95 [8] 2383-2388 (2012).

${ }^{16}$ F. Li, J. Pan, O. Guillon and A. Cocks, "Predicting Sintering Deformation of Ceramics Film Constrained by Rigid Substrate Using Anisotropic Constitutive Law,” Acta Metall., 58 [18] 5980-5988 (2010).

${ }^{17}$ A. Akash and M. J. Mayo, "Pore Growth During Initial-stage Sintering,” J. Am. Ceram. Soc., 82 [11] 2948-2952 (1999).

${ }^{18}$ E.Dörre and H. Hübner, Alumina Processing, Properties, and Applications; pp. 38-40, Springer-Verlag Press, Inc., 1984.

${ }^{19}$ Z. Shen, M. Johnsson, Z. Zhao and M. Nygren, “Spark Plasma Sintering of Alumina,” J. Am. Ceram. Soc., 85 [8] 1921-1927 (2002).

\section{Captions of All Figures}

Fig. 1 The sintering model used in this study (a) geometry overview and (b) local details of the contact neck.

Fig. 2 Time taken for neck radius to reach $\mathrm{a}=25 \% \mathrm{R}$ as a function of the relative diffusivity $\mathcal{D}_{\mathrm{s}} / \mathcal{D}_{\mathrm{gb}}$ for different values of the remote stress $\sigma_{\infty}$.

Fig. 3 Comparison of profiles of triple junction obtained for $\mathcal{D}_{\mathrm{s}} / \mathcal{D}_{\mathrm{gb}}=5$ and 50 respectively at (a) $t / \tau_{g}=10^{-7}$ and (b) $t / \tau_{g}=10^{-5}$

Fig. 4 Neck size as function of time for $\mathcal{D}_{\mathrm{s}} / \mathcal{D}_{\text {gb }}=5$ for different remote stresses of $\sigma_{\infty}$

Fig. 5 Neck size as function of time for $\mathcal{D}_{\mathrm{s}} / \mathcal{D}_{\text {gb }}=50$ for different remote stresses of $\sigma_{\infty}$ Fig. 6 Time taken for shrinkage to reach $\mathrm{w}=1 \% \mathrm{R}$ as function of $\mathcal{D}_{\mathrm{s}} / \mathcal{D}_{\text {gb }}$ for a range of remote stress $\sigma_{\infty}$

This paper was presented at SINTERING 2014, Dresden, Germany. 
Fig. 7 Shrinkage as function of time for $\mathcal{D}_{\mathrm{s}} / \mathcal{D}_{\mathrm{gb}}=5$ and various values of $\sigma_{\infty}$

Fig. 8 Shrinkage as function of time for $\mathcal{D}_{\mathrm{s}} / \mathcal{D}_{\mathrm{gb}}=50$ and various values of $\sigma_{\infty}$

Fig. 9 Effective surface and grain-boundary diffusivities as functions of temperature

Fig. 10 Shrinkage versus neck-size as temperature increases from $600{ }^{\circ} \mathrm{C}$ to $1400{ }^{\circ} \mathrm{C}$ with different heating rate. The applied remote pressure is zero. Data set 1 in Table IV for surface diffusivity was used.

Fig. 11 Shrinkage versus neck-size as temperature increases from $600{ }^{\circ} \mathrm{C}$ to $1400{ }^{\circ} \mathrm{C}$ with different heating rate. The applied remote pressure is $\sigma_{\infty}=-50 \mathrm{MPa}$. Data set 1 in Table IV for surface diffusivity was used.

Fig. 12 Shrinkage versus neck-size as temperature increases from $600{ }^{\circ} \mathrm{C}$ to $1400{ }^{\circ} \mathrm{C}$ with different heating rate. The applied remote pressure is $\sigma_{\infty}=0 \mathrm{MPa}$. Data set 2 in Table IV for surface diffusivity was used.

Fig. 13 Shrinkage versus neck-size as temperature increases from $600{ }^{\circ} \mathrm{C}$ to $1400{ }^{\circ} \mathrm{C}$ with different heating rate. The applied remote pressure is $\sigma_{\infty}=-50 \mathrm{MPa}$. Data set 2 in Table IV for surface diffusivity was used.

\section{Tables}

Table I. Material Data Used in the Numerical Study

\begin{tabular}{|l|c|}
\hline Atomic volume $\boldsymbol{\Omega}$ & $8.47 \times 10^{-30} \mathrm{~m}^{3}$ \\
\hline Specific surface energy $\boldsymbol{\gamma}_{\mathbf{s}}$ & $1.1 \mathrm{~J} / \mathrm{m}^{2}$ \\
\hline Specific grain-boundary energy $\boldsymbol{\gamma}_{\mathbf{g b}}$ & $1.21 \mathrm{~J} / \mathrm{m}^{2}$ \\
\hline Grain-boundary thickness $\times$ pre-exponential grain- & $1.02 \times 10^{-7} \mathrm{~m}^{3} \mathrm{~s}^{-1}$ \\
boundary diffusion coefficient $\boldsymbol{\delta}_{\mathbf{g b}} \mathbf{D}_{\mathbf{g b}} \mathbf{0}$ & $5.3 \times 10^{5} \mathrm{~J} / \mathrm{mol}$ \\
\hline Activation energy for grain-boundary diffusion $\mathbf{Q}_{\mathbf{g b}}$ & $8.314 \mathrm{~J} /(\mathrm{mol} \mathrm{K})$ \\
\hline Gas constant $\mathbf{R}$ & $1.381 \times 10^{-23} \mathrm{~m}^{2} \mathrm{~kg} /\left(\mathrm{s}^{2} \mathrm{~K}\right)$ \\
\hline Boltzmann's constant $\mathbf{k}$ & $130^{\circ}$ \\
\hline Dihedral angle $\boldsymbol{\theta}$ & $10^{-6} \mathrm{~m}$ \\
\hline Initial particle radius $\mathbf{R}$ & \\
\hline
\end{tabular}

This paper was presented at SINTERING 2014, Dresden, Germany. 
Table II. Direction of surface diffusion flux in the vicinity of the triple junction

\begin{tabular}{|c|c|}
\hline$\sigma_{\infty}=0$, & $\begin{array}{c}\text { Direction of surface diffusion flux, }+ \text { for away } \\
\mathcal{D}_{\mathrm{s}} / \mathcal{D}_{\mathrm{gb}}\end{array}$ \\
\hline 5 & from the junction \\
\hline 10 & + \\
\hline 50 & + \\
\hline 100 & - \\
\hline 1000 & - \\
\hline
\end{tabular}

Table III. Values of $\mathrm{D}_{\mathrm{gbo}} \delta_{\mathrm{gb}}$ and $\mathrm{Q}_{g b}$ Used in the Simulation

\begin{tabular}{|c|c|}
\hline $\mathrm{D}_{\mathrm{gb} 0} \delta_{\mathrm{gb}}\left(\mathrm{m}^{3} \mathrm{~s}^{-1}\right)$ & $\mathrm{Q}_{g b}(\mathrm{~J} / \mathrm{mol})$ \\
\hline $8.6 \times 10^{-10}$ & $4.18 \times 10^{5}$ \\
\hline
\end{tabular}

Table IV. Values of $\mathrm{D}_{\mathrm{s} 0}$ and $\mathrm{Q}_{s}$ Used in the Simulation

\begin{tabular}{|c|c|c|}
\hline Set No. & $\mathrm{D}_{\mathrm{s} 0}\left(\mathrm{~m}^{2} \mathrm{~s}^{-1}\right)$ & $\mathrm{Q}_{s}(\mathrm{~J} / \mathrm{mol})$ \\
\hline 1 & $4.8 \times 10^{-6}$ & $2.34 \times 10^{5}$ \\
\hline 2 & $1.15 \times 10^{-3}$ & $2.8 \times 10^{5}$ \\
\hline
\end{tabular}




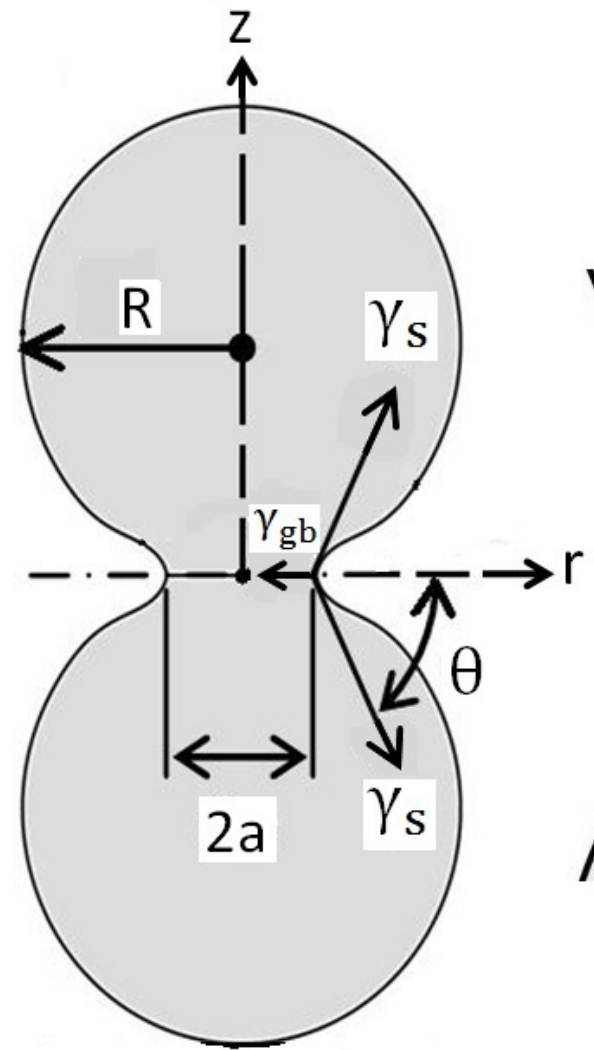

(a) $\sigma_{\infty} \uparrow \uparrow \uparrow \uparrow \uparrow \uparrow$

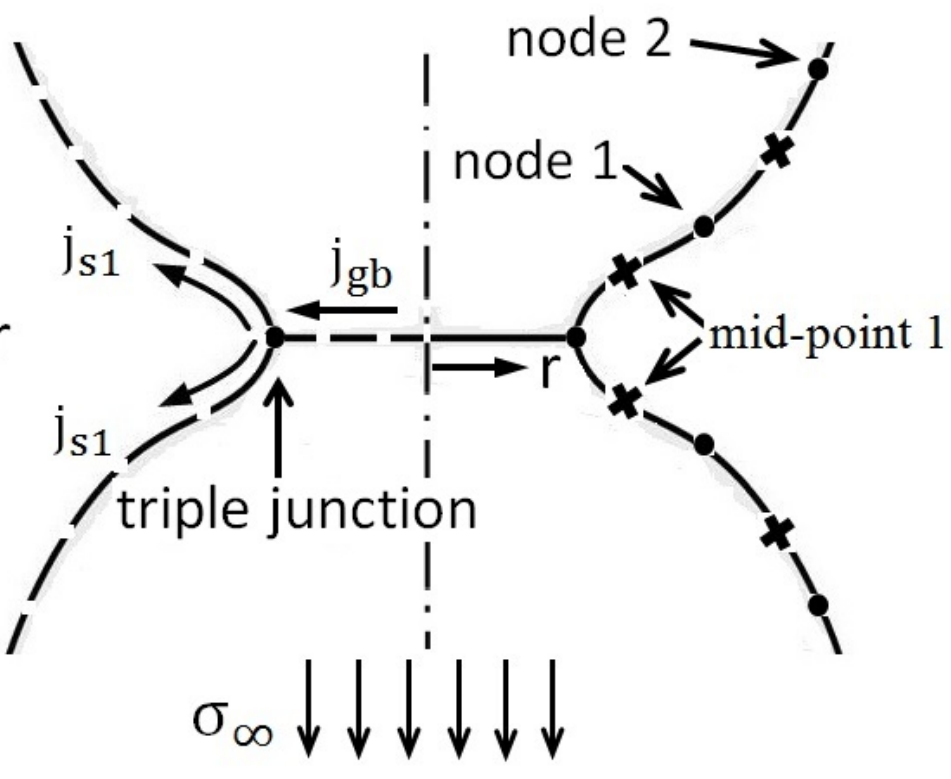

(b)

Fig. 1 The sintering model used in this study (a) geometry overview and (b) local details of the contact neck. 


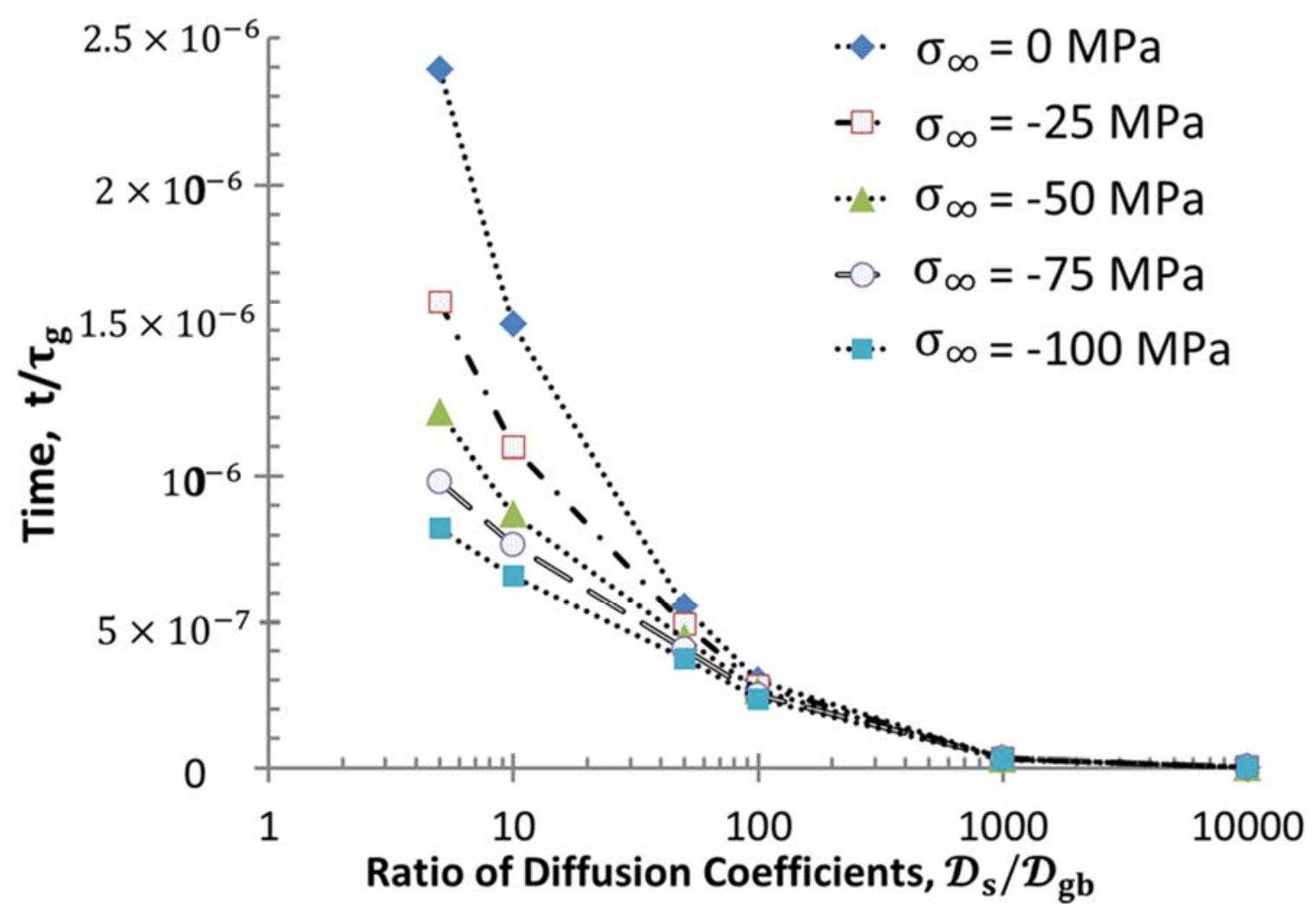

Fig. 2 Time taken for neck radius to reach $\mathrm{a}=25 \% \mathrm{R}$ as a function of the relative diffusivity $\mathcal{D}_{\mathrm{s}} / \mathcal{D}_{\mathrm{gb}}$ for different values of the remote stress $\sigma_{\infty}$. 


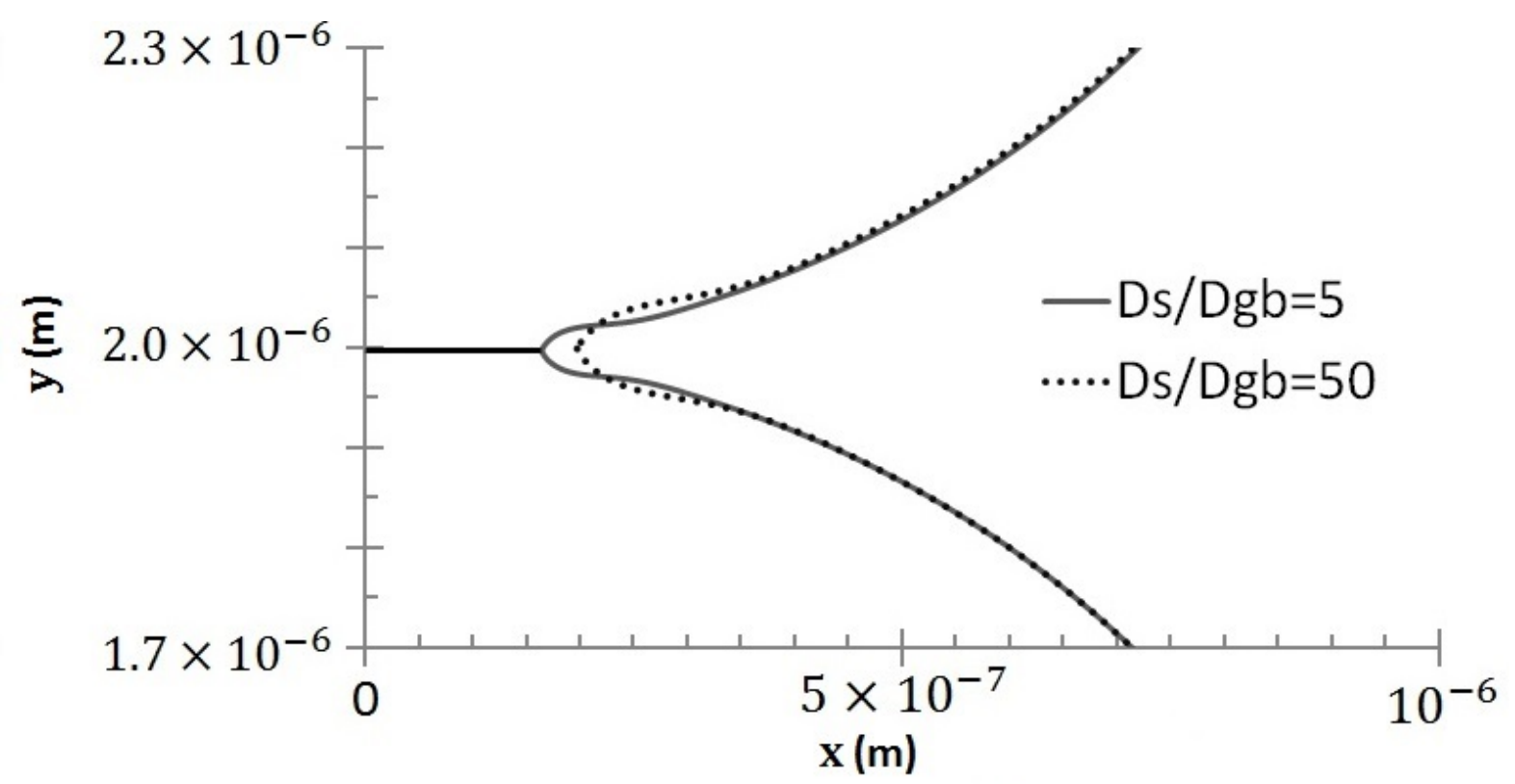

(a) Time, $t / \tau_{g}=10^{-7}$

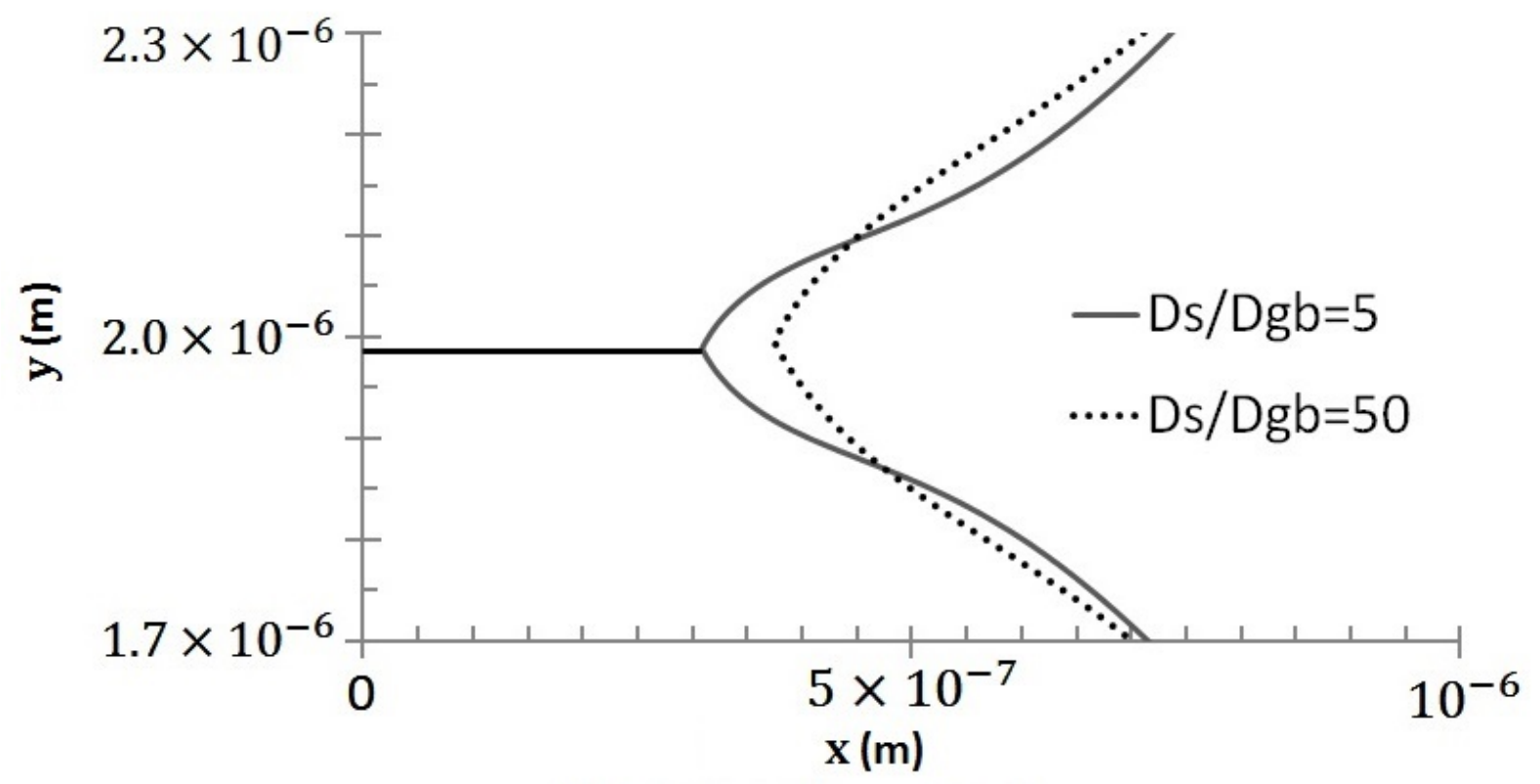

(b) Time, $t / \tau_{g}=10^{-5}$

Fig. 3 Comparison of profiles of triple junction obtained for $\mathcal{D}_{\mathrm{s}} / \mathcal{D}_{\mathrm{gb}}=5$ and 50 respectively at (a) $t / \tau_{g}=10^{-7}$ and (b) $t / \tau_{g}=10^{-5}$ 


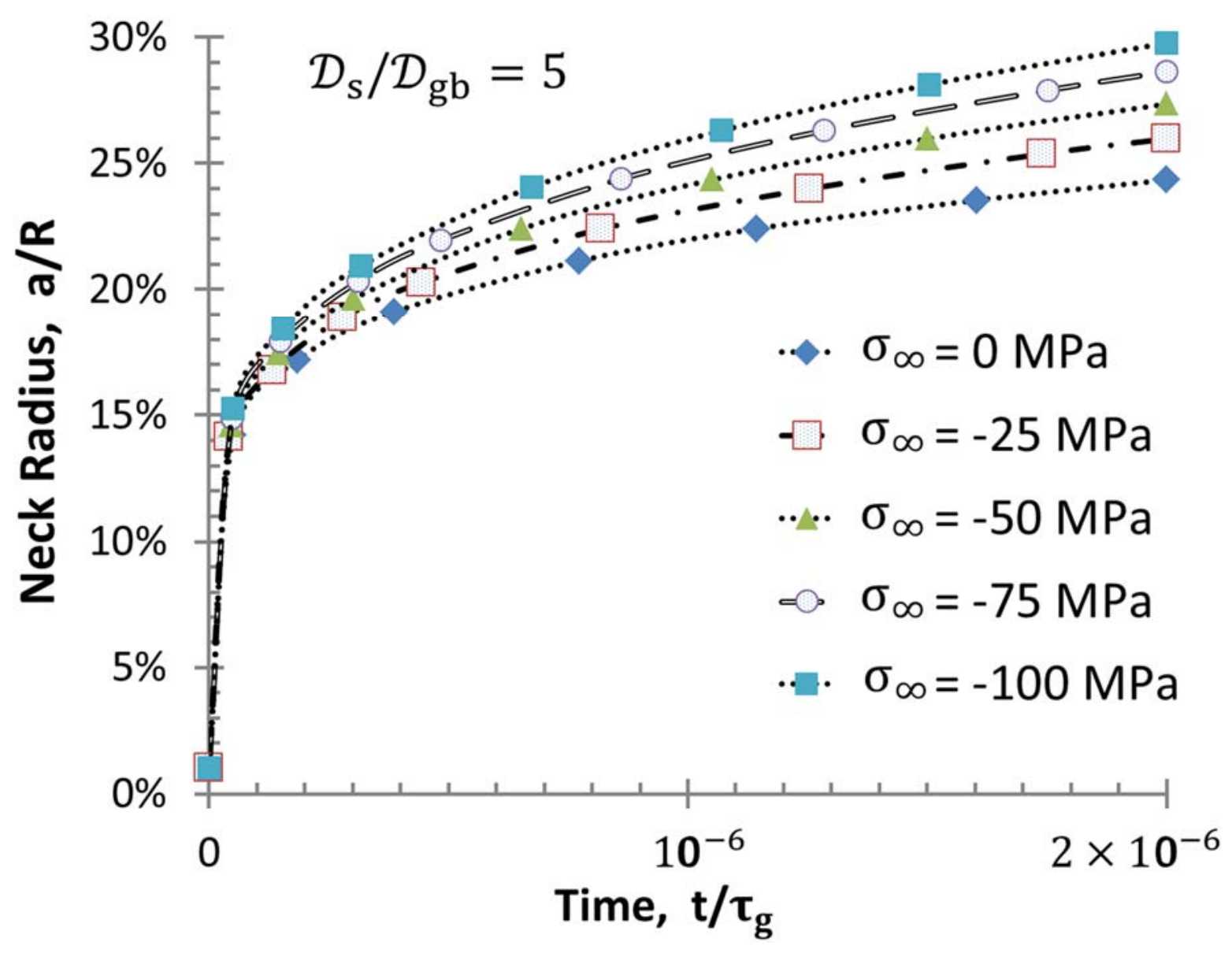

Fig. 4 Neck size as function of time for $\mathcal{D}_{\mathrm{s}} / \mathcal{D}_{\mathrm{gb}}=5$ for different remote stresses of $\sigma_{\infty}$ 


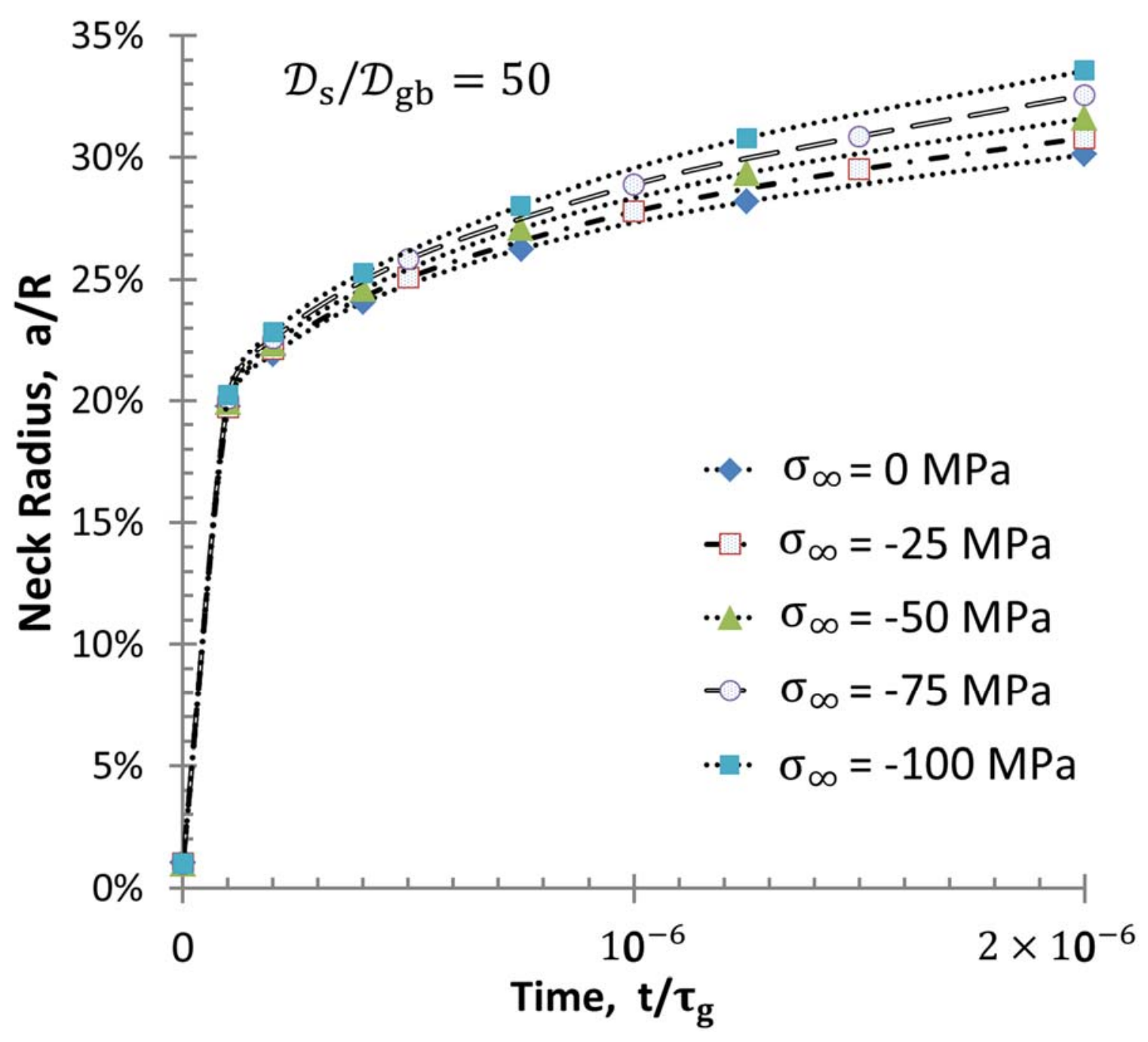

Fig. 5 Neck size as function of time for $\mathcal{D}_{\mathrm{s}} / \mathcal{D}_{\text {gb }}=50$ for different remote stresses of $\sigma_{\infty}$ 


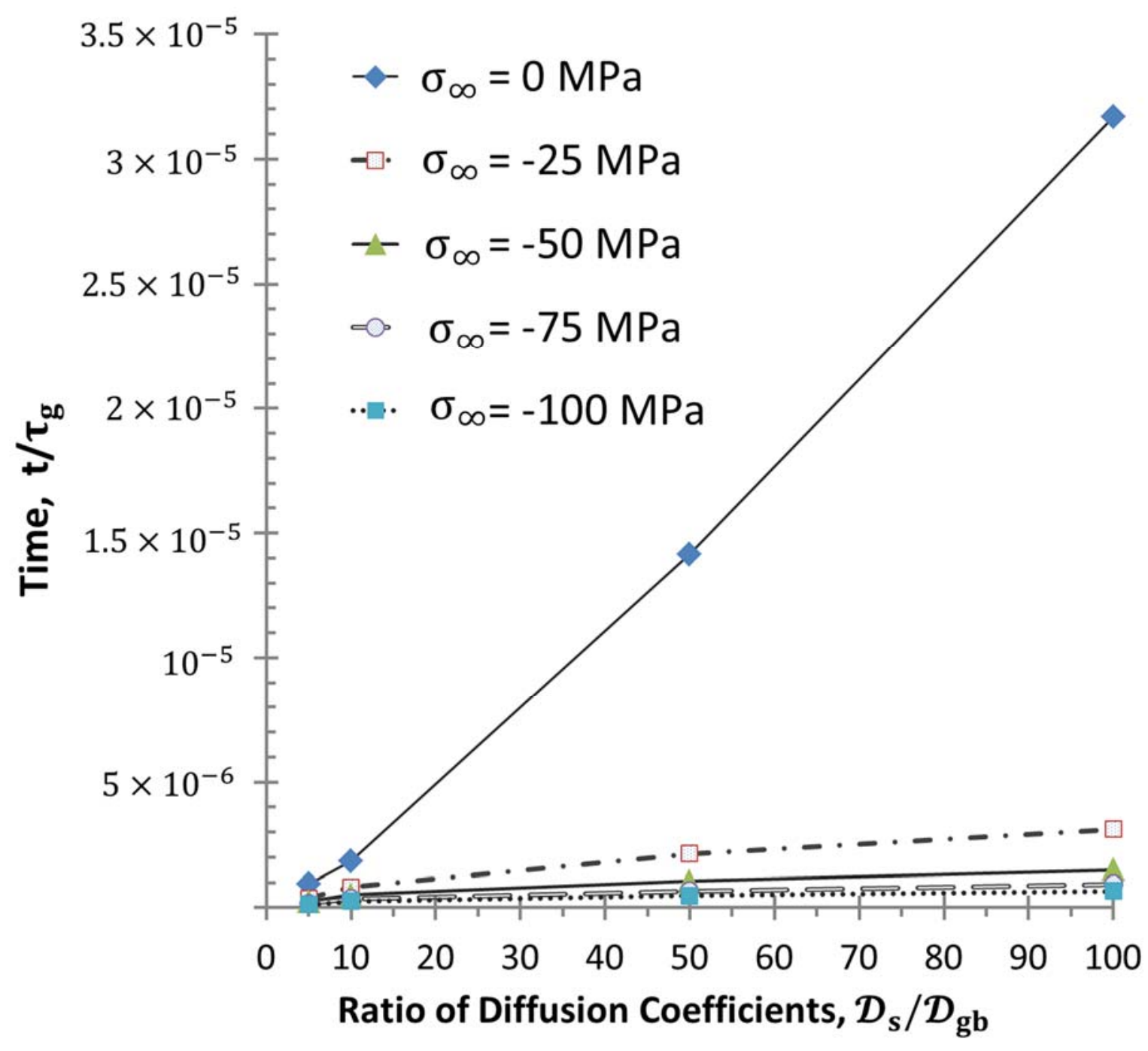

Fig. 6 Time taken for shrinkage to reach $w=1 \%$ as function of $\mathcal{D}_{\mathrm{s}} / \mathcal{D}_{\text {gb }}$ for a range of remote stress $\sigma_{\infty}$ 


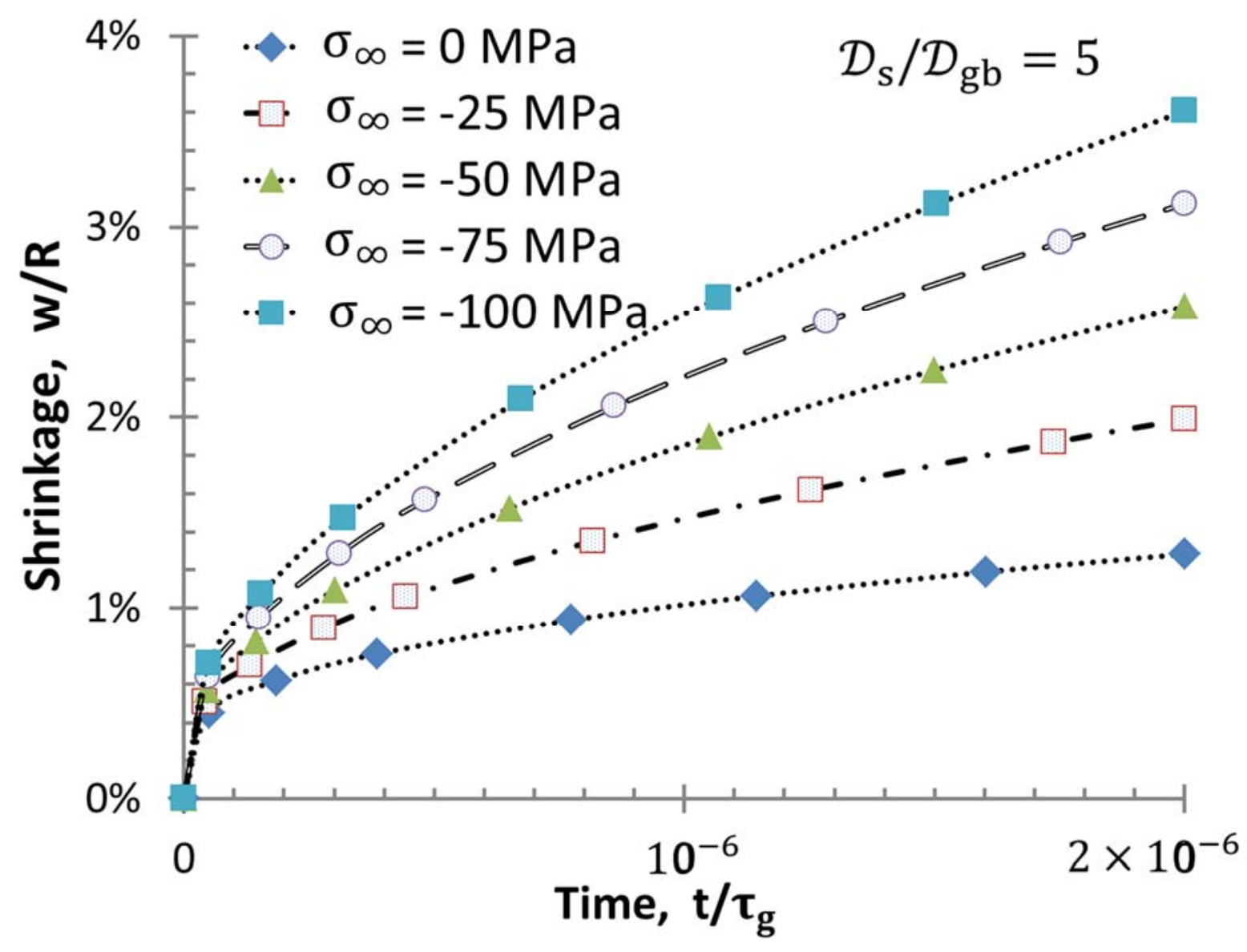

Fig. 7 Shrinkage as function of time for $\mathcal{D}_{\mathrm{s}} / \mathcal{D}_{\mathrm{gb}}=5$ and various values of $\sigma_{\infty}$ 


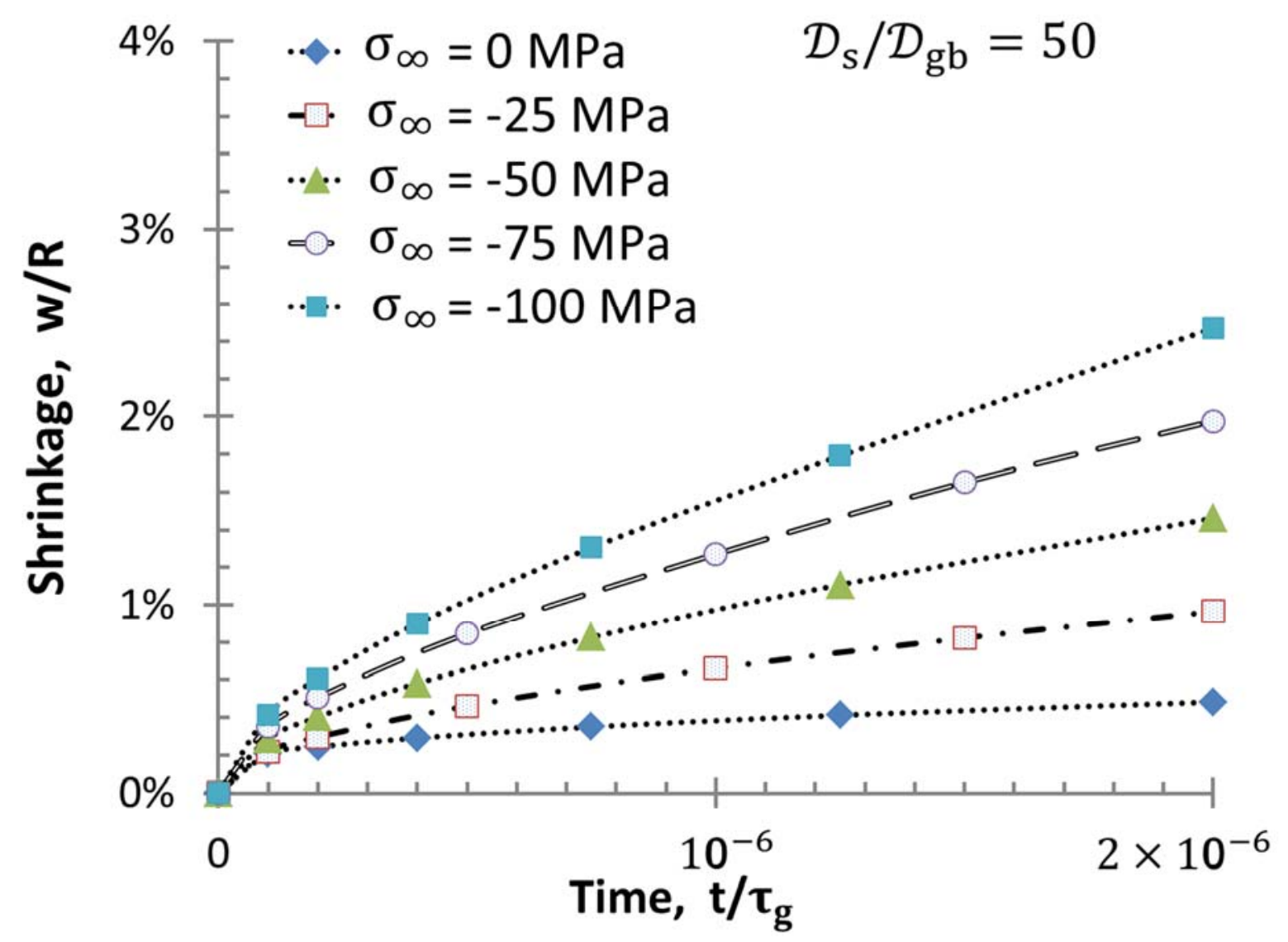

Fig. 8 Shrinkage as function of time for $\mathcal{D}_{\mathrm{s}} / \mathcal{D}_{\mathrm{gb}}=50$ and various values of $\sigma_{\infty}$ 


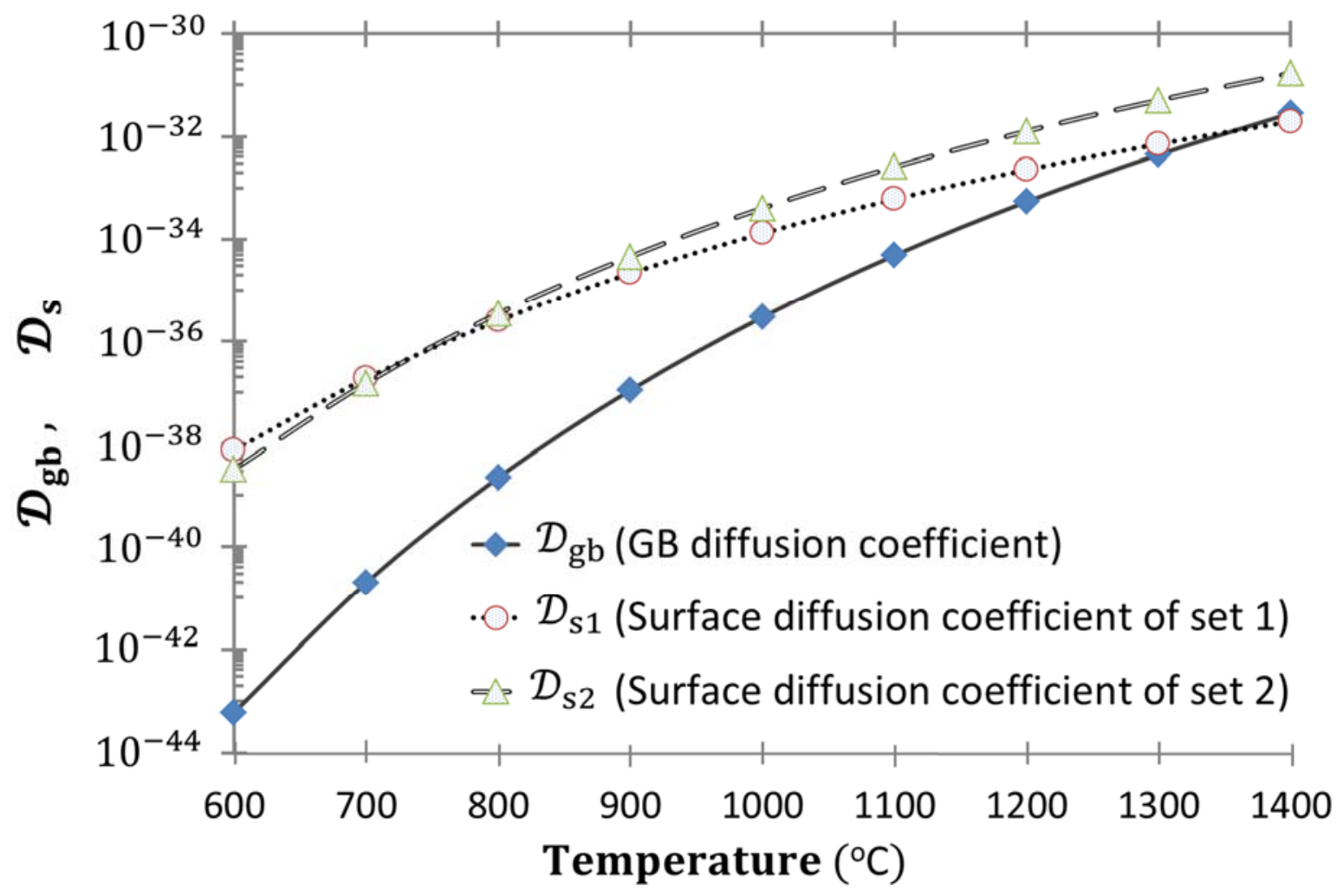

Fig. 9 Effective surface and grain-boundary diffusivities as functions of temperature 


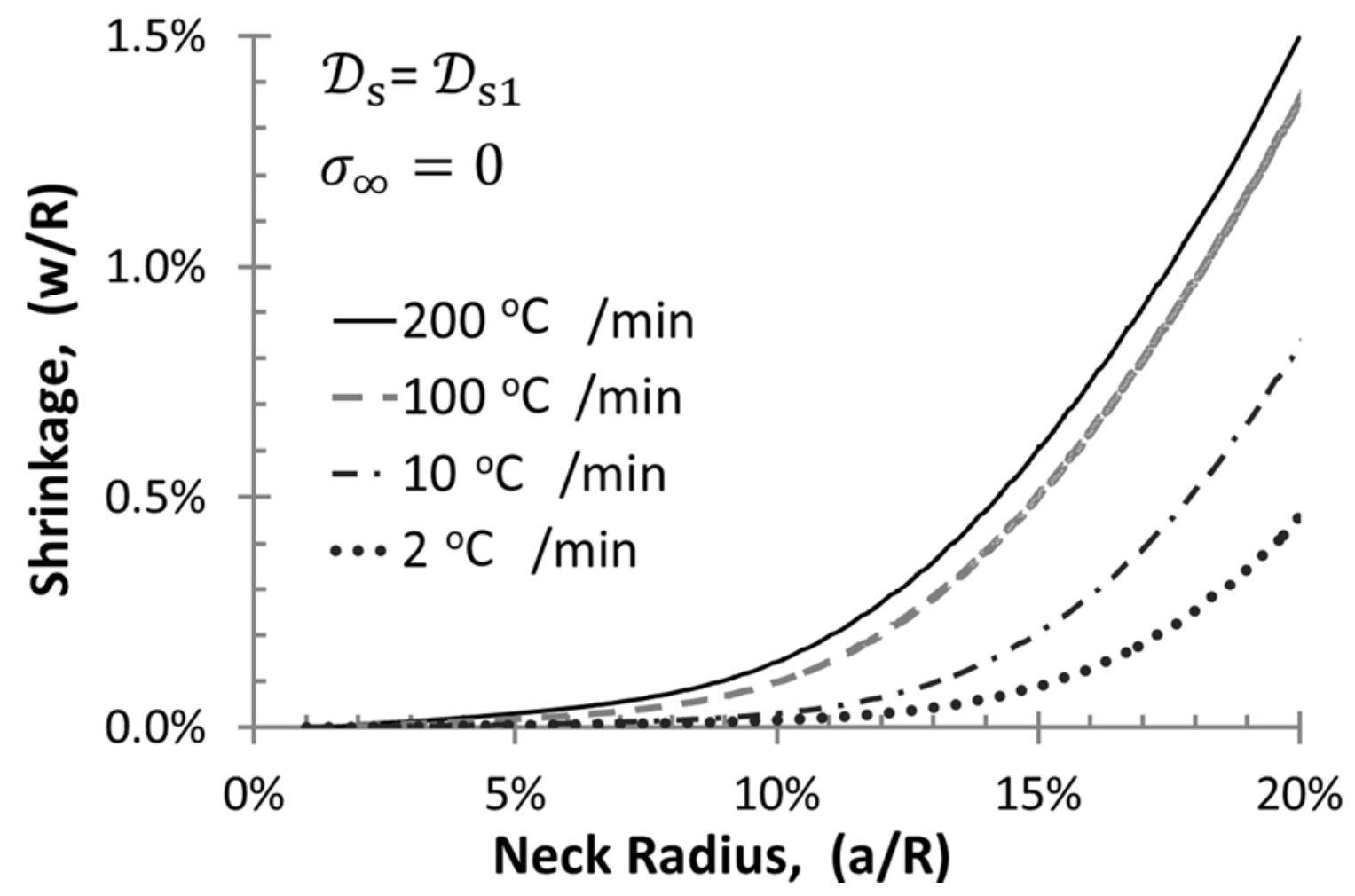

Fig. 10 Shrinkage versus neck-size as temperature increases from $600{ }^{\circ} \mathrm{C}$ to $1400{ }^{\circ} \mathrm{C}$ with different heating rate. The applied remote pressure is zero. Data set 1 in Table IV for surface diffusivity was used. 


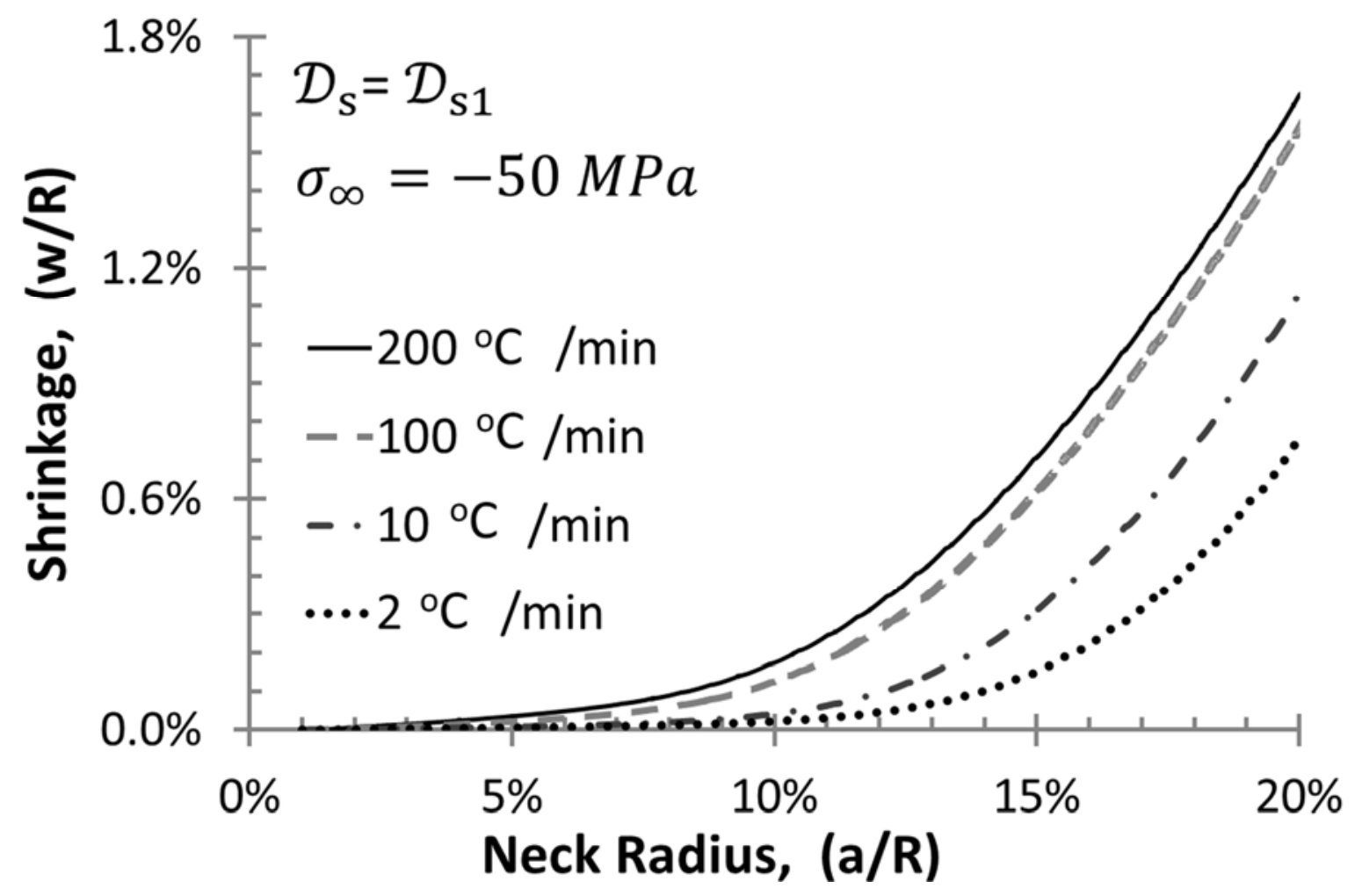

Fig. 11 Shrinkage versus neck-size as temperature increases from $600^{\circ} \mathrm{C}$ to $1400^{\circ} \mathrm{C}$ with different heating rate. The applied remote pressure is $\sigma_{\infty}=-50 \mathrm{MPa}$. Data set 1 in Table IV for surface diffusivity was used. 


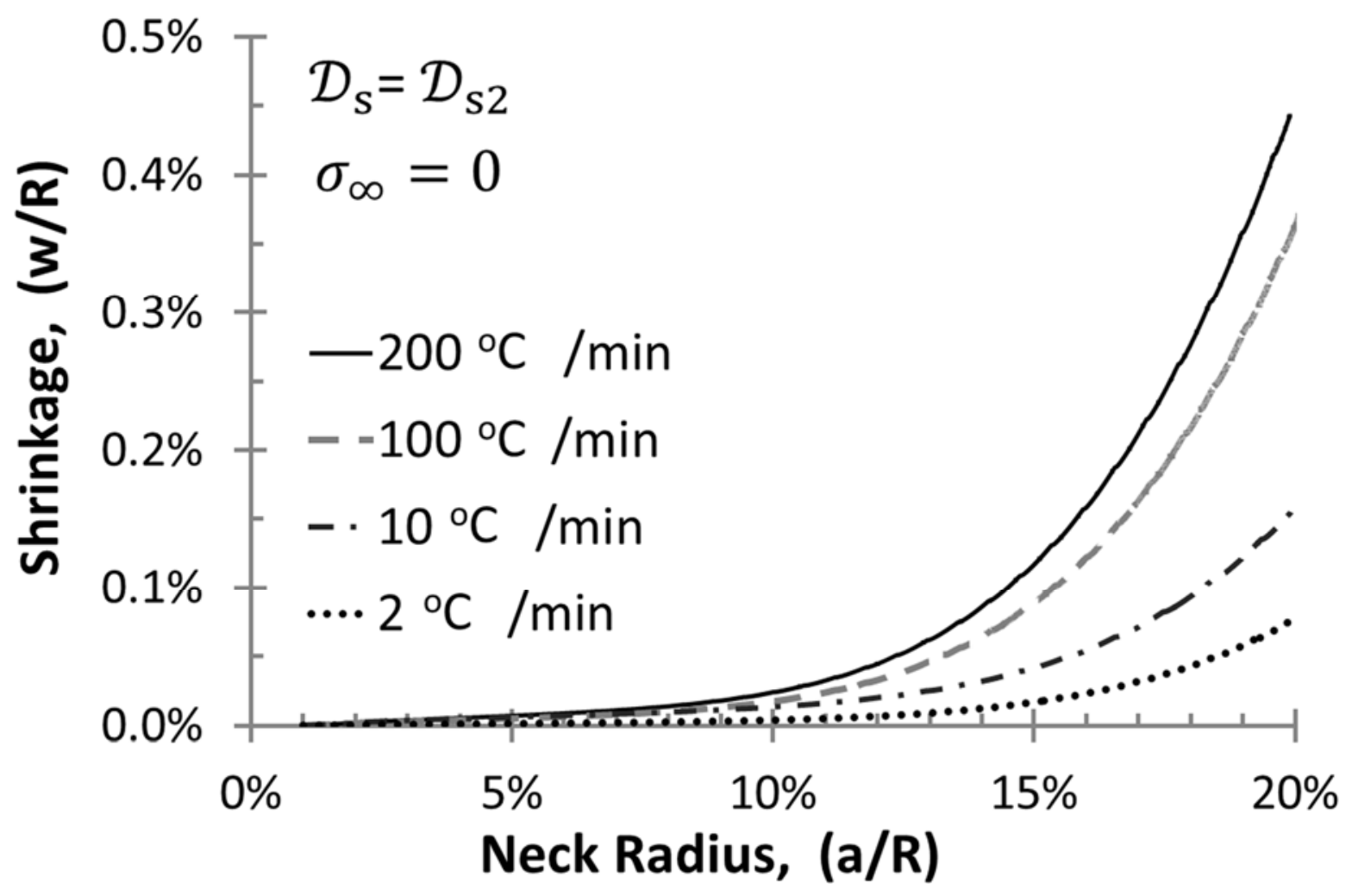

Fig. 12 Shrinkage versus neck-size as temperature increases from $600{ }^{\circ} \mathrm{C}$ to $1400{ }^{\circ} \mathrm{C}$ with different heating rate. The applied remote pressure is $\sigma_{\infty}=0 \mathrm{MPa}$. Data set 2 in Table IV for surface diffusivity was used. 


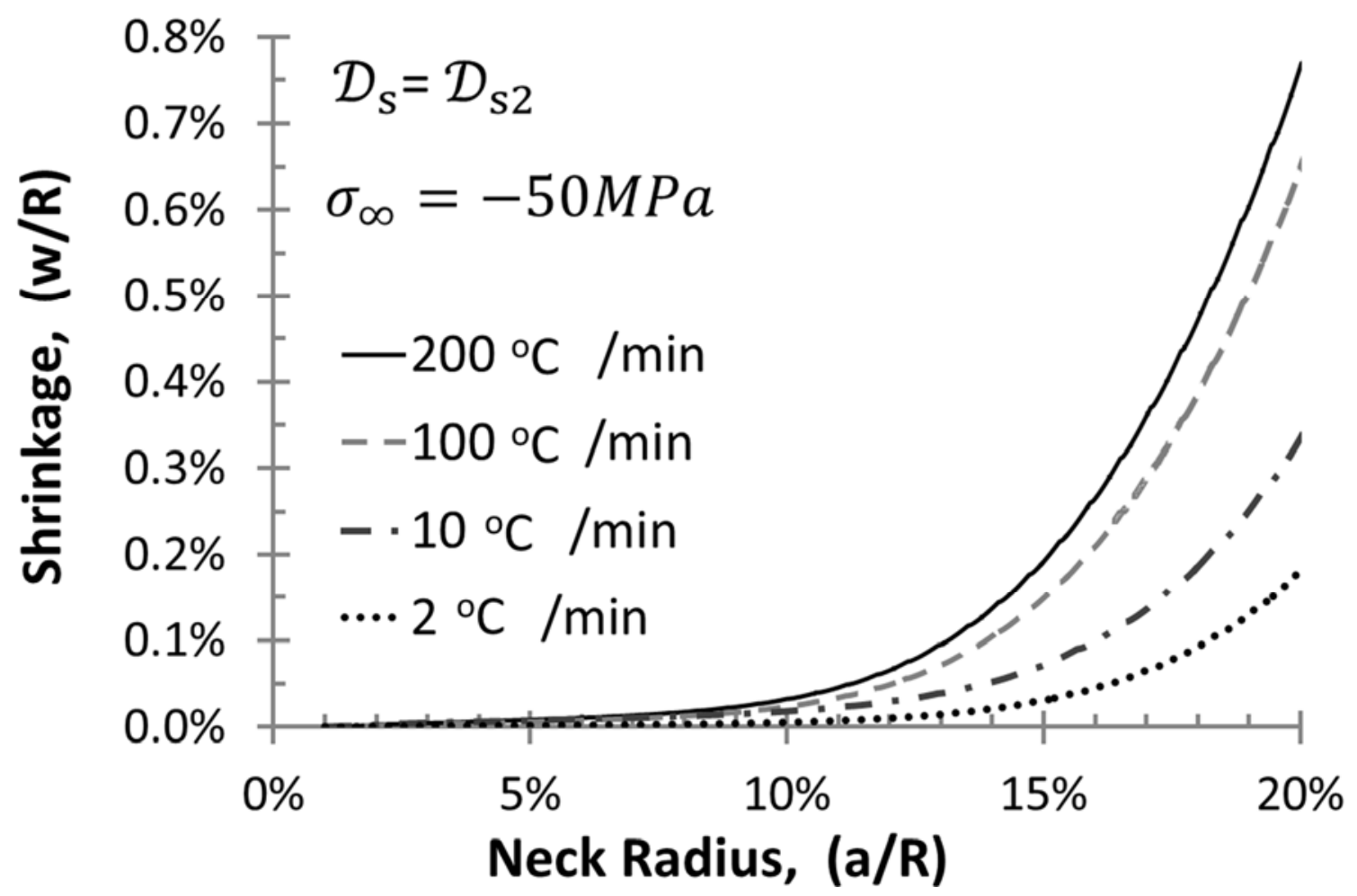

Fig. 13 Shrinkage versus neck-size as temperature increases from $600{ }^{\circ} \mathrm{C}$ to $1400{ }^{\circ} \mathrm{C}$ with different heating rate. The applied remote pressure is $\sigma_{\infty}=-50 M P a$. Data set 2 in Table IV for surface diffusivity was used. 